\title{
Impact of Hepatic Steatosis on Disease-Free Survival in Patients with Non-B Non-C Hepatocellular Carcinoma Undergoing Hepatic Resection.
}

\section{$\operatorname{AUTHOR}(\mathrm{S})$ :}

Nishio, Takahiro; Hatano, Etsuro; Sakurai, Takaki; Taura, Kojiro; Okuno, Masayuki; Kasai, Yosuke; Seo, Satoru; ... Mori, Akira; Kaido, Toshimi; Uemoto, Shinji

\section{CITATION:}

Nishio, Takahiro ... [et al]. Impact of Hepatic Steatosis on Disease-Free Survival in Patients with Non-B Non-C Hepatocellular Carcinoma Undergoing Hepatic Resection.. Annals of surgical oncology 2014, 22(7): 2226-2234

\section{ISSUE DATE:}

2014-11-14

URL:

http://hdl.handle.net/2433/201500

\section{RIGHT:}

The final publication is available at Springer via http://dx.doi.org/10.1245/s10434-0144181-9.; この論文は出版社版でありません。引用の際には出版社版をご確認ご利用くだ さい。; This is not the published version. Please cite only the published version. 
Impact of Hepatic Steatosis on Disease-free Survival in Patients with Non-B Non-C

\section{Hepatocellular Carcinoma Undergoing Hepatic Resection}

Takahiro Nishio, $\mathrm{MD}^{1}$, Etsuro Hatano, MD, $\mathrm{PhD}^{1}$, Takaki Sakurai, MD, $\mathrm{PhD}^{2}$, Kojiro Taura, MD, $\mathrm{PhD}^{1}$, Masayuki Okuno, $\mathrm{MD}^{1}$, Yosuke Kasai, $\mathrm{MD}^{1}$, Satoru Seo, MD, $\mathrm{PhD}^{1}$, Kentaro Yasuchika, MD, $\mathrm{PhD}^{1}$, Akira Mori, $\mathrm{MD}, \mathrm{PhD}^{1}$, Toshimi Kaido, $\mathrm{MD}, \mathrm{PhD}^{1}$, Shinji Uemoto, $\mathrm{MD}, \mathrm{PhD}^{1}$

1. Department of Surgery, Graduate School of Medicine, Kyoto University, Kyoto, Japan

2. Department of Diagnostic pathology, Graduate School of Medicine, Kyoto University, Kyoto, Japan

Corresponding author:

Etsuro Hatano, MD, PhD

Department of Surgery, Graduate School of Medicine, Kyoto University

54 Kawahara-cho, Shogoin, Sakyo-ku, Kyoto, Japan

Tel: +81-75-751-4323, Fax: +81-75-751-4263, E-mail: etsu@kuhp.kyoto-u.ac.jp

Running head:

Non-B Non-C HCC and hepatic steatosis

\section{DISCLOSURES}

The authors declare no conflicts of interest. 


\section{SYNOPSIS}

The prevalence of non-B non-C hepatocellular carcinoma (NBNC HCC) has increased. We categorized its etiology according to alcohol intake and presence of steatosis. Absence of steatosis in hepatic parenchyma had a significant impact on disease-free survival in NBNC HCC patients. 


\section{ABSTRACT}

Background. Although the prevalence of non-B non-C hepatocellular carcinoma (NBNC HCC) has increased, its clinicopathologic characteristics remain unclear.

Methods. We retrospectively analyzed 518 HCC patients who underwent hepatic resection. Hepatitis B surface antigen- and hepatitis C antibody-negative patients were categorized into the NBNC HCC group ( $n=145)$; others were categorized into the hepatitis B or C HCC (BC HCC) group ( $n=373$ ). We subdivided the etiologies of NBNC HCC according to alcohol intake and presence of steatosis.

Results. NBNC HCC was associated with nonalcoholic fatty liver disease (NAFLD) (13.1\%), fatty liver disease (FLD) with moderate alcohol intake (9.0\%), alcoholic liver disease (ALD) (29.7\%), cryptogenic disease (44.1\%), and other known etiologies (4.1\%). The prevalence of obesity, diabetes mellitus, and hypertension was higher and hepatic function was better in the NBNC HCC group, which had significantly larger tumors than the BC HCC group. The entire NBNC HCC group displayed similar overall and disease-free survival as the BC HCC group. Among the subdivisions, NAFLDassociated HCC patients had significantly better disease-free survival than ALDassociated HCC and BC HCC patients. Microvascular invasion (hazard ratio [HR], 2.30; 95\% confidence interval [CI], 1.33-3.96) and steatosis area $<5 \%$ of non-cancerous region (HR, 2.13; 95\% CI, 1.21-3.93) were associated with disease-free survival in NBNC HCC patients.

Conclusions. The prognosis of NBNC HCC was similar to that of BC HCC. Among NBNC HCC patients, NAFLD-associated HCC patients had a relatively low recurrence risk. Absence of steatosis in hepatic parenchyma had a significant impact on diseasefree survival in NBNC HCC patients. 
Hepatocellular carcinoma (HCC) is the fifth most common cancer and the third most frequent cause of cancer-related death worldwide. ${ }^{1,2}$ Liver cirrhosis is the primary risk factor for HCC development, ${ }^{3,4}$ which is the long-term result of chronic hepatitis B (HBV) or hepatitis C virus (HCV) infection or chronic alcohol consumption. ${ }^{5-7}$ Recently, the prevalence of hepatitis B surface antigen (HBsAg)- and hepatitis C antibody (HCVAb)-negative HCC_-also termed non-B non-C HCC (NBNC HCC)—has rapidly increased $^{8-10}$, whereas that of HBV- or HCV-related HCC has decreased owing to antiviral therapy. ${ }^{11}$

The etiology of NBNC HCC is multifactorial and remains unorganized. In addition to chronic alcohol consumption, nonalcoholic fatty liver disease (NAFLD) and metabolic syndrome-related factors (i.e., obesity, insulin resistance, diabetes mellitus, dyslipidemia, and hypertension) are considered risk factors for NBNC HCC. ${ }^{10-16}$ According to the nationwide survey of non-B non-C liver cirrhosis in Japan at the $15^{\text {th }}$ General Meeting of the Japan Society of Hepatology, nonalcoholic steatohepatitis (NASH, 14.5\%), alcoholic liver disease (ALD, 55.1\%), fatty liver disease (FLD) with moderate alcohol consumption (intake, 20-70 g ethanol/day, 2.5\%), primary biliary cirrhosis $(8.0 \%)$, other biliary cirrhosis $(0.8 \%)$, autoimmune hepatitis $(6.8 \%)$, metabolic disease $(0.6 \%)$, congestive disease $(0.8 \%)$, parasitic disease $(0.2 \%)$, other known etiology $(0.2 \%)$, and unknown etiology $(10.5 \%)$ were identified as the causes of non-B non-C liver cirrhosis. ${ }^{17}$ Chronic alcohol consumption, obesity-associated metabolic syndrome, and their common pathological consequences characterized by steatosis are considered essential etiological factors for categorizing NBNC HCC. Studies have examined the clinicopathologic characteristics of NBNC HCC; however, none systematically categorized NBNC HCC patients based on etiology and pathology of hepatic parenchyma. 
These etiological factors may be closely associated with tumor characteristics, preoperative hepatic functional reserve, and the resulting surgical outcomes of NBNC HCC patients. We investigated the clinicopathologic features and prognostic factors of NBNC HCC patients who underwent hepatic resection. We categorized their etiology based on alcohol consumption, metabolic syndrome prevalence, and pathology of underlying parenchymal change, especially focusing on presence of steatosis, which is a key etiological criterion of NBNC HCC.

\section{PATIENTS AND METHODS}

\section{Patients}

Between January 2000 and December 2011, 688 HCC patients underwent initial hepatic resection at our institution. We excluded 170 patients because the pathological findings of fibrosis and steatosis in the parenchyma were not precisely evaluated. The remaining 518 patients were divided into 2 groups: patients negative for HBsAg and HCVAb were categorized into the NBNC HCC group ( $n=145)$; others were included in the HBV- or HCV-related HCC (BC HCC) group (n=373). NBNC HCC patients were further categorized according to the etiological criteria of the nationwide survey of nonB non-C liver cirrhosis in Japan at the $15^{\text {th }}$ General Meeting of the Japan Society of Hepatology ${ }^{17}$ as: (i) ALD, with heavy alcohol consumption (ethanol intake $>70$ g/day); (ii) NAFLD, characterized by histological fatty liver without chronic alcohol consumption (ethanol intake $\leq 20 \mathrm{~g} /$ day); (iii) FLD, also characterized by histological fatty liver and moderate alcohol consumption (ethanol intake, 20-70 g/day); (iv) other known etiology (e.g., autoimmune hepatitis, cholestatic diseases, or other chronic liver diseases); and (v) cryptogenic diseases. Among the conventional cryptogenic HCC cases, we tentatively established the following criteria of non-steatotic and metabolic 
syndrome-associated HCC (Non-Steatotic Met HCC): daily ethanol intake <70 g/day; absence of the pathological steatosis; and prevalence of one or more of metabolic syndrome-associated risk factors. The definitions of the risk criteria for metabolic syndrome were based on the international criteria ${ }^{16,18}$ with slight modification to suit the Japanese criteria as follows: obesity (body mass index $>25 \mathrm{~kg} / \mathrm{m}^{2}$ ); diabetes mellitus (fasting glucose $\geq 126 \mathrm{mg} / \mathrm{dL}$ or $\mathrm{HbA1c} \geq 6.5 \%$ ); dyslipidemia (increased triglycelides $\geq 150 \mathrm{mg} / \mathrm{dL}$ or reduced HDL cholesterol $<40 \mathrm{mg} / \mathrm{dL}$ in men or $<50 \mathrm{mg} / \mathrm{dL}$ in women); and hypertension (systolic $\geq 130$ and/or diastolic $\geq 85 \mathrm{mmHg}$ ). Drug treatment for diabetes, dyslipidemia, or hypertension was an alternative indicator.

This study was in accordance with the ethical guidelines for epidemiological research in Japan and was approved by the Ethics Committee of the Kyoto University Graduate School and Faculty of Medicine (approval code: E2029).

\section{Data collection}

History of alcohol intake was collected using an interview-sheet, and the data were confirmed by the participants' families, doctors, and nurses. The indocyanine green retention rate at 15 min (ICG R15) was measured as a marker of preoperative liver function. Serum alpha-fetoprotein (AFP) and des-gamma-carboxy prothrombin (DCP) levels were measured before surgical treatment. Surgical complications were classified according to the Clavien-Dindo classification. ${ }^{19,20}$ In addition to pathological tumor characteristics, the pathological findings of non-cancerous liver tissue were evaluated. Presence of steatosis was defined according to the amount of surface area involved by fatty infiltration: none, $<5 \%, 5-33 \%$, and $>33 \%$. Even in cases with minimum steatosis (steatosis area $<5 \%$ ), patients were diagnosed as positive for steatosis. NBNC HCC patients with steatosis were further assessed according to their 
NAFLD activity score (NAS) ${ }^{21}$. Liver fibrosis progression was recorded using the Metavir score ${ }^{22}$; F4 was defined as pathological cirrhosis.

\section{Statistical analysis}

Categorical variables were presented as numbers and percentages. Continuous variables were presented as mean \pm standard deviation. Differences in categorical variables were analyzed using the chi-squared or Fisher's exact test; continuous variables were compared using Mann-Whitney's U test. Median survival and 95\% confidence intervals (95\% CIs) were determined using the Kaplan-Meier method; groups were compared using the log-rank test. Variables that were associated with survival at $p<0.10$ in univariate survival analysis were included in a multivariate Cox regression analysis, and hazard ratios (HRs) and 95\% CIs were calculated. In all analyses, $p<0.05$ was considered statistically significant. JMP 10 software (SAS institute Inc. Cary, NC) was used in all statistical analyses.

\section{RESULTS}

Etiology of NBNC HCC

The proportion of NBNC HCC patients who underwent surgical resection has recently increased (Figure 1). The etiology of NBNC HCC was multifactorial and complex, and we conventionally categorized patients according to the alcohol intake level and presence of pathological steatosis (Figure 2). ALD ( $n=43,29.7 \%)$ was a major cause of disease, followed by NAFLD $(n=19,13.1 \%)$ and FLD ( $n=13,9.0 \%)$, which were commonly characterized by steatosis without alcohol intake and steatosis with moderate alcohol intake, respectively. Despite the complexity of the etiological criteria, $>40 \%$ of cases of NBNC HCC were categorized as cryptogenic HCC ( $n=64,44.1 \%)$. In 
such conventional cryptogenic cases, we tentatively established the criteria of NonSteatotic Met HCC: absence of heavy alcohol intake; absence of steatosis; and presence of metabolic syndrome-associated risk factors, including obesity, diabetes mellitus, dyslipidemia, or hypertension (Figure 2). Non-Steatotic Met HCC patients ( $n=43$ ) accounted for $29.7 \%$ of all NBNC HCC cases and represented more than half of all cases originally categorized as cryptogenic HCC. Among the 6 patients with other known etiologies (4.1\%), 4 had autoimmune hepatitis; 1, primary biliary cirrhosis; and 1, Budd-Chiari syndrome.

\section{Clinicopathologic characteristics of NBNC HCC}

The clinicopathologic characteristics of the NBNC HCC and BC HCC groups were examined (Table 1). Age, BMI, and the prevalence of diabetes mellitus and hypertension were significantly higher among NBNC HCC patients than among BC HCC patients. The NBNC HCC group had a greater proportion of patients with low alcohol intake (ethanol intake $\leq 20 \mathrm{~g} /$ day) and more patients who were heavy drinkers (ethanol intake $>70 \mathrm{~g} /$ day), reflecting the etiological criteria of ALD and NAFLD. The hepatic functional reserve based on the Child-Pugh classification and ICG R15 and other liver damage indicators (e.g., transaminase and albumin levels, prothrombin activity, and platelet count), were better in the NBNC HCC group. Larger tumor size and higher serum DCP levels were preoperative tumor characteristics of the NBNC HCC group, whereas serum AFP levels were lower among these patients. The number of tumors, presence of microvascular invasion, and TNM stage were not significantly different between the 2 groups. Moreover, NBNC HCC patients were less likely to have fibrosis and more likely to display steatosis than BC HCC patients. The NBNC HCC group had more patients who underwent major hepatectomy; thereby, the surgical time 
was longer in this group. Surgical blood loss and the rate of operative complications did not differ significantly between the groups.

Furthermore, we examined the characteristics of each subdivision of NBNC HCC group (Supplemental Table 1). Cryptogenic HCC patients proved to have more advanced tumors, and they were characterized by higher DCP levels, and a higher rate of poorly differentiated tumor and microvascular invasion than Non-Steatotic Met HCC patients.

We additionally examined the NAS of NAFLD-associated HCC patients (Supplemental Figure 1) as NAFLD ranges from simple steatosis to NASH. The diagnosis of NAFLD was made according to NAS $\geq 3$, including possible NASH cases. The characteristics of these patients were assessed (Supplemental Table 2). The high NAS group had more advanced tumors than the low NAS group, but liver function and the prevalence of cirrhosis did not differ significantly. BMI and the rate of obesity were significantly higher in the low NAS group.

\section{Survival analysis of patients with NBNC HCC and BC HCC}

The median overall survival times of the NBNC HCC $(n=145)$ and BC HCC groups ( $\mathrm{n}=373)$ were 78.5 (95\% CI, 59.9-85.5) and 72.4 months (95\% CI, 58.4-81.4), respectively ( $p=0.436$ ). The median disease-free survival times among patients in the NBNC HCC $(n=126)$ and BC HCC groups $(n=304)$ who underwent curative resection were 24.5 (95\% CI, 19.5-33.8) and 20.5 months (95\% CI, 17.6-25.3), respectively ( $p=0.2953)$. Prognosis in the 2 groups did not differ significantly. Among the various etiologies, the overall and disease-free survival rates of NAFLD-associated HCC patients were better than those of ALD-associated HCC and BC HCC patients (see Supplemental Figures 2 and 3). On the other hand, the prognosis of Non-Steatotic Met 
HCC and cryptogenic HCC was similarly as poor as that of ALD-associated HCC or BC HCC.

Table 2 presents the results of univariate and multivariate analyses of the disease-free survival of NBNC HCC patients who underwent curative resection. Alcohol intake levels and the prevalence of obesity, diabetes, or dyslipidemia were not associated with disease-free survival. Although several studies reported that serum hepatitis B core (HBc) antibody positivity, also termed “occult hepatitis B virus infection,” is a risk factor for NBNC HCC, ${ }^{8,23,24}$ we did not identify significant involvement of $\mathrm{HBC}$ antibody positivity in the risk of NBNC HCC recurrence. Multivariate analysis revealed that the presence of microvascular invasion and steatosis area $<5 \%$ of non-cancerous region were significantly associated with disease-free survival after curative resection (Table 2). However, liver cirrhosis did not significantly affect the recurrence risk among NBNC HCC patients.

Impact of steatosis on disease-free survival

Liver cirrhosis is a powerful risk factor for HCC development, and it was consistent with the disease-free survival of BC HCC patients in our study (Supplemental Figure 4). In NBNC HCC patients, liver cirrhosis did not affect the recurrence risk (Supplemental Figure 5). On the other hand, the absence of steatosis was associated with the disease-free survival of NBNC HCC patients (Supplemental Figure 6), but not with that of BC HCC patients (Supplemental Figure 7). The relationship between steatosis area and disease-free survival is shown in Figure 3 and suggests that a lesser extent of steatosis led to a higher risk of HCC progression.

To assess the possibility that the absence of steatosis might be a consequence of liver fibrosis progression, thus explaining the poor prognosis of NBNC HCC patients, 
we analyzed the correlations between steatosis and liver fibrosis. Liver fibrosis was scored in NBNC HCC patients with steatosis area $\geq 5 \%(n=39)$ as: $F 0,25.6 \%$; $F 1$, 20.5\%; F2, 18.0\%; F3, 10.3\%; and F4, 25.6\%. Conversely, liver fibrosis in NBNC HCC patients with steatosis area $<5 \%(n=95)$ was categorized as: F0, 20.7\%; F1, 34.0\%; F2, 13.2\%; F3, 12.3\%; and F4, 19.8\%. No correlations between the extent of steatosis and the progression of fibrosis were found.

\section{DISCUSSION}

Recently, the prevalence of NBNC HCC has rapidly increased. ${ }^{11,25}$ NAFLD and NASH, the major causes of NBNC HCC, are associated with metabolic syndrome risk factors. ${ }^{13,16,26-28}$ The epidemic rise in obesity and metabolic syndrome might explain the increase in the NBNC HCC prevalence in Japan and Western countries. Our study showed that the proportion of NBNC HCC patients who underwent surgical resection has also increased. The prevalence of obesity, diabetes mellitus, and hypertension was higher among NBNC HCC patients than among BC HCC patients, suggesting that obesity and metabolic syndrome are important risk factors for NBNC HCC. The tumor sizes of the NBNC HCC group were significantly larger than those of the BC HCC group. As their hepatic function was relatively preserved, NBNC HCC patients were frequently diagnosed incidentally or symptomatically without follow-up for their liver damage, possibly resulting in tumor progression at the time of detection. The higher serum DCP levels of NBNC HCC patients might be related with the larger tumor size of these patients, whereas the lower serum AFP levels implied less progression of liver damage. ${ }^{29-32}$ Major hepatectomy was frequently performed among NBNC HCC patients, owing to the large tumor size and good hepatic function reserve. Our survival analysis after surgical treatment revealed no significant difference in prognosis between the 2 
groups, suggesting that the better hepatic functional reserve of NBNC HCC patients is offset by their more advanced tumors.

Multivariate survival analysis revealed that microvascular invasion and smaller steatosis area in the parenchyma were associated with the disease-free survival of NBNC HCC patients. Interestingly, pathological steatosis had a stronger effect on the recurrence risk after curative resection than pathological liver fibrosis, which is the strongest predisposing factor for HCC development. ${ }^{1,3,4}$ Our study indicated that the absence of steatosis, but not its promotion, was involved in higher recurrence risk. Patients with NAFLD-associated HCC had a significantly lower recurrence risk than patients with ALD-associated HCC and BC HCC. However, the recurrence risks of Non-Steatotic Met HCC patients, who did not fulfill the pathological criteria for NAFLD but exhibited metabolic syndrome-associated risk factors, were not different from those of ALD-associated HCC and BC HCC patients. Absence of steatosis in the NBNC HCC background might indicate the presence of somewhat chronic liver damage not characterized by the promotion of liver fibrosis.

This study has several potential limitations. First, self-reports on alcohol intake might be inaccurate. However, we attempted to overcome such a limitation by using a questionnaire form and verifying patient reports with doctors, nurses, and families. Second, the presence of steatosis was based on focal observation of the non-cancerous region of the resected liver tissue and it did not necessarily represent the characteristics of the entire underlying parenchymal changes. Third, the prevalence of obesity and metabolic syndrome in the Japanese population differs from that of Western countries, and so we had to partly modify the international diagnostic criteria of obesity and metabolic syndrome.

In conclusion, chronic alcohol consumption, metabolic syndrome, and their 
common pathological consequence (i.e., steatosis) are risk factors for NBNC HCC development. Absence of steatosis had a stronger impact on disease-free survival than liver fibrosis in NBNC HCC patients. In postoperative surveillance and lifestyle education, we should devote more attention to such patients with NBNC HCC and those who lack steatosis in the parenchyma. 


\section{REFERENCES}

1. Llovet JM, Burroughs A, Bruix J. Hepatocellular carcinoma. Lancet. 2003;362(9399):1907-1917.

2. Jemal A, Siegel R, Xu J, Ward E. Cancer statistics, 2010. CA Cancer J Clin. 2010;60(5):277-300.

3. Zaman SN, Melia WM, Johnson RD, Portmann BC, Johnson PJ, Williams R. Risk factors in development of hepatocellular carcinoma in cirrhosis: prospective study of 613 patients. Lancet. 1985;1(8442):1357-1360.

4. Johnson PJ, Williams R. Cirrhosis and the aetiology of hepatocellular carcinoma. J Hepatol. 1987;4(1):140-147.

5. Bruix J, Sherman M, Llovet JM, et al. Clinical management of hepatocellular carcinoma. Conclusions of the Barcelona-2000 EASL conference. European Association for the Study of the Liver. J Hepatol. 2001;35(3):421-430.

6. Ikeda K, Saitoh S, Koida I, et al. A multivariate analysis of risk factors for hepatocellular carcinogenesis: a prospective observation of 795 patients with viral and alcoholic cirrhosis. Hepatology. 1993;18(1):47-53.

7. Tsukuma H, Hiyama T, Tanaka S, et al. Risk factors for hepatocellular carcinoma among patients with chronic liver disease. $N$ Engl $J$ Med. 1993;328(25):1797-1801. 
8. Abe H, Yoshizawa K, Kitahara T, Aizawa R, Matsuoka M, Aizawa Y. Etiology of non-B non-C hepatocellular carcinoma in the eastern district of Tokyo. $J$ Gastroenterol. 2008;43(12):967-974.

9. Tanaka H, Imai Y, Hiramatsu N, et al. Declining incidence of hepatocellular carcinoma in Osaka, Japan, from 1990 to 2003. Ann Intern Med. 2008;148(11):820-826.

10. Nagaoki Y, Hyogo H, Aikata H, et al. Recent trend of clinical features in patients with hepatocellular carcinoma. Hepatol Res. 2012;42(4):368-375.

11. Utsunomiya T, Shimada M. Molecular characteristics of non-cancerous liver tissue in non-B non-C hepatocellular carcinoma. Hepatol Res. 2011;41(8):711721.

12. Starley BQ, Calcagno CJ, Harrison SA. Nonalcoholic fatty liver disease and hepatocellular carcinoma: a weighty connection. Hepatology. 2010;51(5):18201832.

13. Bugianesi E, Leone N, Vanni E, et al. Expanding the natural history of nonalcoholic steatohepatitis: from cryptogenic cirrhosis to hepatocellular carcinoma. Gastroenterology. 2002;123(1):134-140.

14. Nair S, Mason A, Eason J, Loss G, Perrillo RP. Is obesity an independent risk factor for hepatocellular carcinoma in cirrhosis? Hepatology. 2002;36(1):150- 
155.

15. Siegel AB, Zhu AX. Metabolic syndrome and hepatocellular carcinoma: two growing epidemics with a potential link. Cancer. 2009;115(24):5651-5661.

16. Cauchy F, Zalinski S, Dokmak S, et al. Surgical treatment of hepatocellular carcinoma associated with the metabolic syndrome. Br J Surg. 2013;100(1):113121.

17. Suzuki Y, Ohtake T, Nishiguchi S, et al. Survey of non-B, non-C liver cirrhosis in Japan. Hepatol Res. 2012.

18. Eckel RH, Alberti KG, Grundy SM, Zimmet PZ. The metabolic syndrome. Lancet. 2010;375(9710):181-183.

19. Dindo D, Demartines N, Clavien PA. Classification of surgical complications: a new proposal with evaluation in a cohort of 6336 patients and results of a survey. Ann Surg. 2004;240(2):205-213.

20. Clavien PA, Barkun J, de Oliveira ML, et al. The Clavien-Dindo classification of surgical complications: five-year experience. Ann Surg. 2009;250(2):187-196.

21. Kleiner DE, Brunt EM, Van Natta M, et al. Design and validation of a histological scoring system for nonalcoholic fatty liver disease. Hepatology. 2005;41(6):1313-1321.

22. Intraobserver and interobserver variations in liver biopsy interpretation in 
patients with chronic hepatitis C. The French METAVIR Cooperative Study Group. Hepatology. 1994;20(1 Pt 1):15-20.

23. Kondo R, Nakashima O, Sata M, et al. Pathological characteristics of patients who develop hepatocellular carcinoma with negative results of both serous hepatitis B surface antigen and hepatitis C virus antibody. Hepatol Res. 2013.

24. Yano Y, Yamashita F, Sumie S, et al. Clinical features of hepatocellular carcinoma seronegative for both HBsAg and anti-HCV antibody but positive for anti-HBc antibody in Japan. Am J Gastroenterol. 2002;97(1):156-161.

25. Michitaka K, Nishiguchi S, Aoyagi Y, et al. Etiology of liver cirrhosis in Japan: a nationwide survey. J Gastroenterol. 2010;45(1):86-94.

26. Hamaguchi M, Kojima $\mathrm{T}$, Takeda $\mathrm{N}$, et al. The metabolic syndrome as a predictor of nonalcoholic fatty liver disease. Ann Intern Med. 2005;143(10):722728.

27. Bedogni G, Miglioli L, Masutti F, Tiribelli C, Marchesini G, Bellentani S. Prevalence of and risk factors for nonalcoholic fatty liver disease: the Dionysos nutrition and liver study. Hepatology. 2005;42(1):44-52.

28. Marchesini G, Bugianesi E, Forlani G, et al. Nonalcoholic fatty liver, steatohepatitis, and the metabolic syndrome. Hepatology. 2003;37(4):917-923.

29. Lok AS, Sterling RK, Everhart JE, et al. Des-gamma-carboxy prothrombin and 
alpha-fetoprotein as biomarkers for the early detection of hepatocellular carcinoma. Gastroenterology. 2010;138(2):493-502.

30. Marrero JA, Feng Z, Wang Y, et al. Alpha-fetoprotein, des-gamma carboxyprothrombin, and lectin-bound alpha-fetoprotein in early hepatocellular carcinoma. Gastroenterology. 2009;137(1):110-118.

31. Li C, Zhang Z, Zhang P, Liu J. Diagnostic accuracy of des-gamma-carboxy prothrombin versus $\alpha$-fetoprotein for hepatocellular carcinoma: A systematic review. Hepatol Res. 2013.

32. Taura N, Ichikawa T, Miyaaki H, et al. Frequency of elevated biomarkers in patients with cryptogenic hepatocellular carcinoma. Med Sci Monit. 2013;19:742-750. 


\section{FIGURE LEGENDS}

Figure 1 The time trend of the proportion of HCC patients who underwent hepatic resection.

Figure 2 Etiological criteria of NBNC HCC. The vertical axis indicates the alcohol intake amount; the horizontal axis indicates the presence of steatosis. Each etiological category is enclosed in a rectangle. Non-steatotic and metabolic syndrome-associated HCC (Non-Steatotic Met HCC) is shown as a gray-fill pattern. Cases of other known etiology are not shown.

Figure 3 Comparison of disease-free survival rates according to the steatosis area of non-cancerous region in NBNC HCC patients who underwent curative resection. Median survival time (MST), 95\% CI, and disease-free survival rate at 5 years (5-year DFS rate) are shown below the graph. Blanks in MST and the upper limit of 95\% CI represent unreached. 


\section{TABLES}

Table 1 Clinicopathologic characteristics of NBNC HCC and BC HCC patients

\begin{tabular}{|c|c|c|c|c|c|c|}
\hline & & \multicolumn{2}{|c|}{ NBNC HCC $(\mathrm{n}=145)$} & \multicolumn{2}{|c|}{ BC HCC $(n=373)$} & \multirow[t]{2}{*}{$p$ value } \\
\hline Clinical backgrounds & & & & & & \\
\hline \multirow[t]{2}{*}{ Gender } & Male & 120 & $(82.8 \%)$ & 283 & $(75.9 \%)$ & 0.0844 \\
\hline & Female & 25 & $(17.2 \%)$ & 90 & $(24.1 \%)$ & \\
\hline Age, yr & & 69 & \pm 9.3 & 64 & \pm 10.6 & $<0.0001$ \\
\hline BMI, kg/ m² & & 23.7 & \pm 3.8 & 23.0 & \pm 3.2 & 0.0465 \\
\hline Obesity, + & & 42 & $(28.0 \%)$ & 86 & $(23.1 \%)$ & 0.166 \\
\hline Diabetes, + & & 62 & $(42.7 \%)$ & 78 & $(20.9 \%)$ & $<0.0001$ \\
\hline Dyslipidemia, + & & 20 & $(13.8 \%)$ & 31 & $(8.3 \%)$ & 0.068 \\
\hline Hypertension, + & & 68 & $(46.9 \%)$ & 138 & $(37.0 \%)$ & 0.0397 \\
\hline Ethanol intake $>70$ g/day & & 43 & (29.9\%) & 31 & $(8.5 \%)$ & $<0.0001$ \\
\hline Ethanol intake $<20$ g/day & & 74 & (51.4\%) & 265 & $(72.8 \%)$ & $<0.0001$ \\
\hline \multicolumn{7}{|l|}{ Liver functions } \\
\hline ICG R15, \% & & 15.3 & \pm 8.5 & 18 & \pm 11.6 & 0.0200 \\
\hline \multirow[t]{2}{*}{ Child-Pugh classification } & A & 138 & $(95.2 \%)$ & 332 & $(89.0 \%)$ & 0.0212 \\
\hline & B & 7 & $(4.8 \%)$ & 41 & $(11.0 \%)$ & \\
\hline AST, IU/l & & 43.5 & \pm 27.7 & 59.6 & \pm 39.7 & $<0.0001$ \\
\hline ALT, IU/l & & 41.1 & \pm 34.9 & 56.5 & \pm 44.9 & $<0.0001$ \\
\hline
\end{tabular}


Platelet count, $x 10^{3} / \mu 1$

Prothrombin activity, \%

Total bilirubin, mg/dl

Albumin, mg/dl

Tumor characteristics

$\mathrm{AFP}, \mathrm{ng} / \mathrm{ml}$

DCP, $\mathrm{mAU} / \mathrm{ml}$

Tumor size, cm

Number of tumors

Tumor differentiation

Well

Moderate

Poor

Microvascular invasion, +

Lymph node involvement, +

TNM Stage

I, II

III, IV

Steatosis, +

Cirrhosis, +

Solitary

\section{Underlying parenchymal changes}

175.6

$\pm 88.5$

86.1

0.84

4.00

$\pm 0.37$

$\pm 14.3$

$\pm 0.50$

0.86

0.0808

3.88

$\pm 0.49$

0.0069

91

(62.8\%)

239

(64.1\%)

0.7799

54

(37.2\%)

134

(35.9\%)

10

(7.0\%)

57

(15.8\%)

0.0071

103

(72.5\%)

215

(59.9\%)

29

(20.4\%)

87

(24.2\%)

57

(39.3\%)

136

(36.5\%)

0.5479

1

(0.7\%)

4

(1.1\%)

0.6791

100

(69.0\%)

286

(76.7\%)

0.0742

45

(31.0\%)

87

(23.3\%)
50

31

(21.4\%)
$<0.0001$

$<0.0001$ 


\begin{tabular}{|c|c|c|c|c|c|c|}
\hline \multicolumn{7}{|l|}{ Surgical factors } \\
\hline \multirow[t]{3}{*}{ Surgical procedure } & $\geq$ Lobectomy & 66 & $(45.5 \%)$ & 139 & $(37.3 \%)$ & 0.0351 \\
\hline & Segmentectomy & 32 & (22.1\%) & 67 & $(17.9 \%)$ & \\
\hline & $\leq$ Subsegmentectomy & 47 & (32.4\%) & 167 & $(44.8 \%)$ & \\
\hline Surgical time, min & & 384.7 & \pm 126.1 & 362.5 & \pm 140.4 & 0.0190 \\
\hline Surgical blood loss, ml & & 1913.2 & \pm 2883.5 & 1682.7 & \pm 2874.8 & 0.4435 \\
\hline \multirow[t]{2}{*}{ Surgical complication } & Grade $\geq$ III & 27 & $(18.6 \%)$ & 83 & $(22.2 \%)$ & 0.3595 \\
\hline & No, Grade $\leq$ II & 118 & $(81.4 \%)$ & 290 & $(77.8 \%)$ & \\
\hline \multirow[t]{2}{*}{ Curativity } & Curative resection & 126 & (86.9\%) & 304 & (81.5\%) & 0.1337 \\
\hline & Not curative resection & 19 & (13.1\%) & 69 & $(18.5 \%)$ & \\
\hline
\end{tabular}

Data represent the mean \pm standard deviation or the number of patients.

NBNC HCC: non-B non-C hepatocellular carcinoma

BC HCC: hepatitis B virus- or hepatitis C virus-related hepatocellular carcinoma

BMI: body mass index

ICG R15: indocyanine green retention rate at 15 minutes

AST: asparatate aminotransferase

ALT: alanine aminotransferase

AFP: alpha-fetoprotein

DCP: des-gamma-carboxy prothrombin 
Table 2 Disease-free survival analysis in NBNC HCC patients

\begin{tabular}{|c|c|c|c|c|c|c|c|}
\hline & \multicolumn{4}{|c|}{ Univariate analysis } & \multicolumn{3}{|c|}{ Multivariate analysis } \\
\hline & & $\begin{array}{c}\text { MST } \\
\text { (months) }\end{array}$ & $95 \%$ CI & $p$ value & Hazard ratio & $95 \%$ CI & $p$ value \\
\hline \multirow[t]{2}{*}{ Gender } & Male & 26.5 & $(22.3-45.1)$ & 0.0331 & 0.84 & $(0.47-1.60)$ & 0.5978 \\
\hline & Female & 16.1 & $(5.5-20.9)$ & & reference & & \\
\hline \multirow[t]{2}{*}{ Age } & $\geq 65 \mathrm{yr}$ & 23.7 & $(19.5-31.4)$ & 0.8122 & & & \\
\hline & $<65 \mathrm{yr}$ & 27.5 & $(9.7-71.7)$ & & & & \\
\hline \multirow[t]{2}{*}{ Obesity } & + & 39.6 & (18.4- .) & 0.1837 & & & \\
\hline & - & 23.0 & $(15.3-28.6)$ & & & & \\
\hline \multirow[t]{2}{*}{ Ethanol intake } & $\geq 70$ g/day & 24.1 & $(11.7-33.8)$ & 0.2035 & & & \\
\hline & $<70$ g/day & 26.5 & $(18.4-71.7)$ & & & & \\
\hline \multirow[t]{2}{*}{ Ethanol intake } & $\geq 20$ g/day & 25.2 & $(13.1-33.8)$ & 0.1504 & & & \\
\hline & $<20$ g/day & 27.5 & $(16.3-102.7)$ & & & & \\
\hline \multirow[t]{2}{*}{ Diabetes } & + & 28.6 & $(14.8-71.7)$ & 0.25 & & & \\
\hline & - & 21.9 & $(16.3-29.1)$ & & & & \\
\hline \multirow[t]{2}{*}{ Dyslipidemia } & + & 45.8 & $(12.4-)$. & 0.1812 & & & \\
\hline & - & 22.5 & $(18.0-28.6)$ & & & & \\
\hline \multirow[t]{2}{*}{ Hypertension } & + & 20.9 & $(12.7-25.5)$ & 0.0361 & 1.38 & $(0.82-2.34)$ & 0.2228 \\
\hline & - & 31.4 & $(19.8-102.7)$ & & reference & & \\
\hline $\mathrm{HBcAb}$ & + & 27.5 & (13.4-64.7) & 0.3021 & & & \\
\hline
\end{tabular}




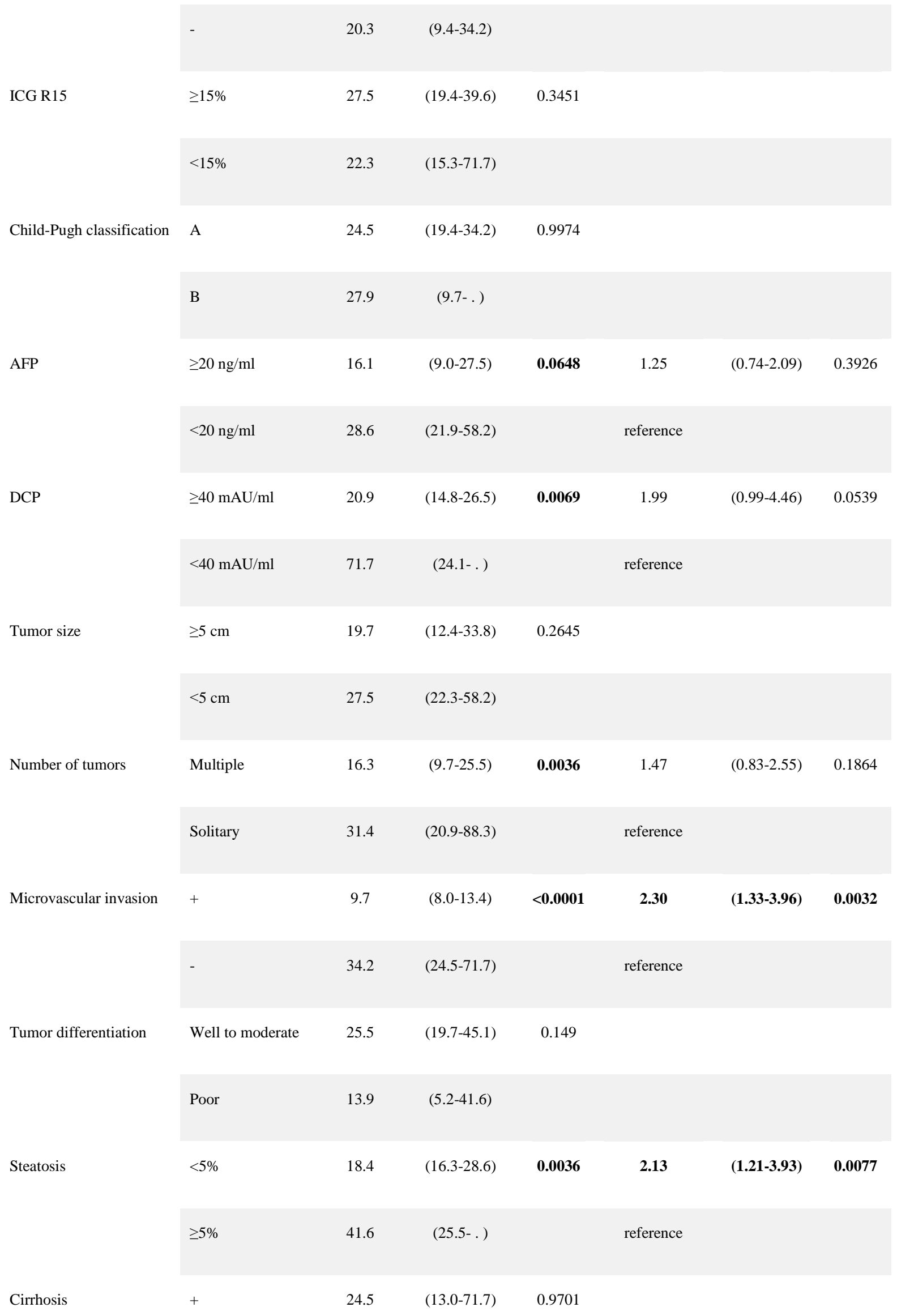




\begin{tabular}{|c|c|c|c|c|c|c|c|}
\hline \multirow{3}{*}{ TNM Stage } & - & 24.1 & \multicolumn{5}{|l|}{$(16.3-39.6)$} \\
\hline & I, II & 31.4 & $(22.5-64.7)$ & $<0.0001$ & 0.72 & $(0.39-1.35)$ & 0.3119 \\
\hline & III, IV & 9.7 & $(6.7-19.7)$ & & reference & & \\
\hline \multirow[t]{2}{*}{ Surgical procedure } & $\geq$ Lobectomy & 15.3 & $(9.5-25.2)$ & 0.0256 & 0.71 & $(0.40-1.26)$ & 0.2454 \\
\hline & $<$ Segmentectomy & 34.2 & $(22.5-58.2)$ & & reference & & \\
\hline \multirow[t]{2}{*}{ Surgical time } & $\geq 360 \mathrm{~min}$ & 25.5 & $(15.3-45.1)$ & 0.7861 & & & \\
\hline & $<360 \min$ & 22.3 & $(16.1-41.6)$ & & & & \\
\hline \multirow[t]{2}{*}{ Surgical blood loss } & $\geq 1000 \mathrm{ml}$ & 24.5 & $(15.3-34.2)$ & 0.6795 & & & \\
\hline & $<1000 \mathrm{ml}$ & 24.1 & $(18.4-45.8)$ & & & & \\
\hline \multirow[t]{2}{*}{ Surgical complication } & Grade $\geq$ III & 26.5 & $(6.7-34.2)$ & 0.7051 & & & \\
\hline & No, Grade $\leq$ II & 24.1 & $(18.4-41.6)$ & & & & \\
\hline
\end{tabular}

Curative resection cases of NBNC HCC $(n=126)$ were analyzed.

Blanks in the upper limit of 95\% CI represent unreached.

NBNC HCC: non-B non-C hepatocellular carcinoma

MST: median survival time

95\% CI: 95\% confidence intervals

HBcAb: hepatitis B core antibody

ICG R15: indocyanine green retention rate at 15 minutes

AFP: alpha-fetoprotein

DCP: des-gamma-carboxy prothrombin 
Figure 1

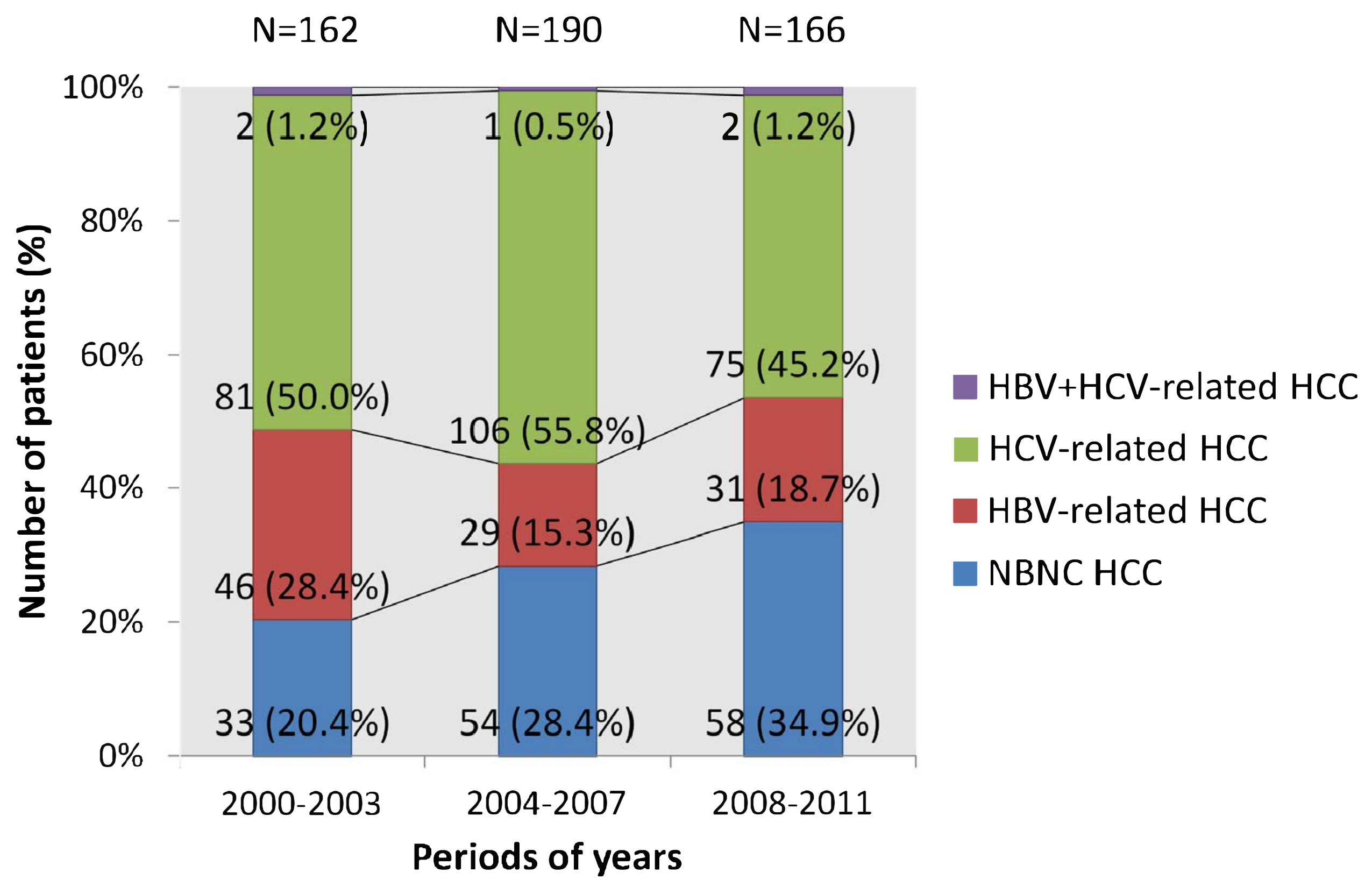


Figure 2

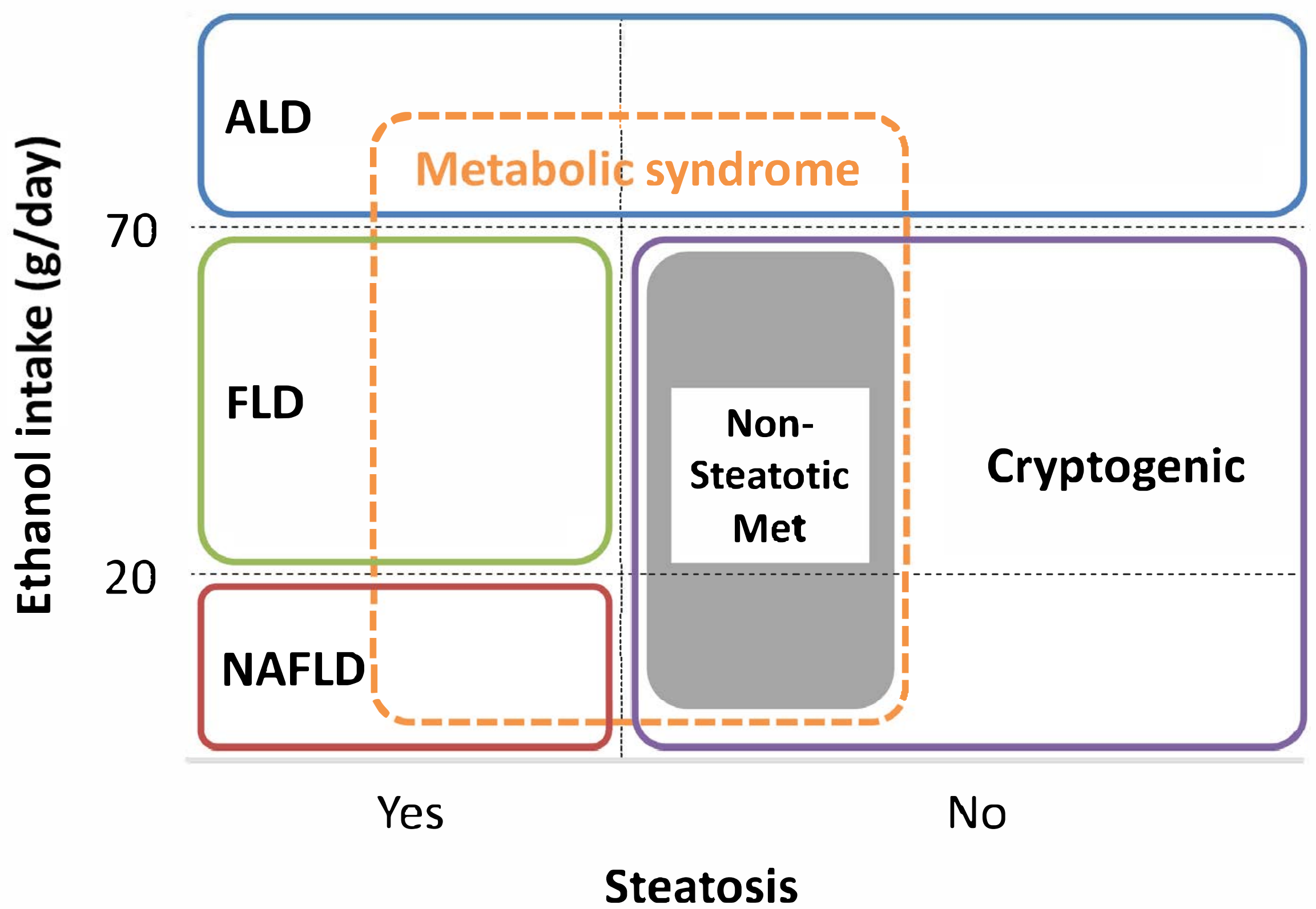




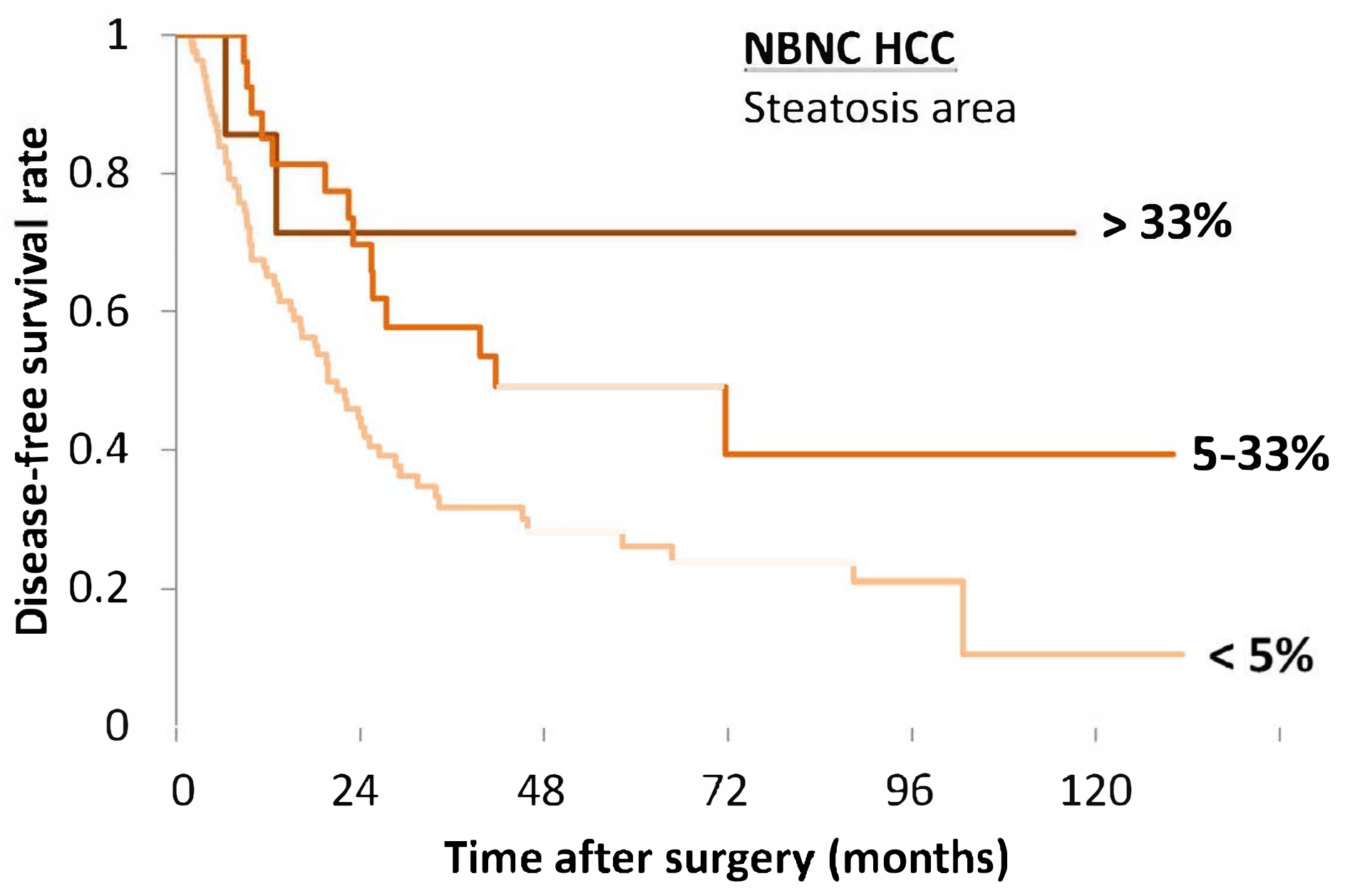

Figure 3

Steatosis area

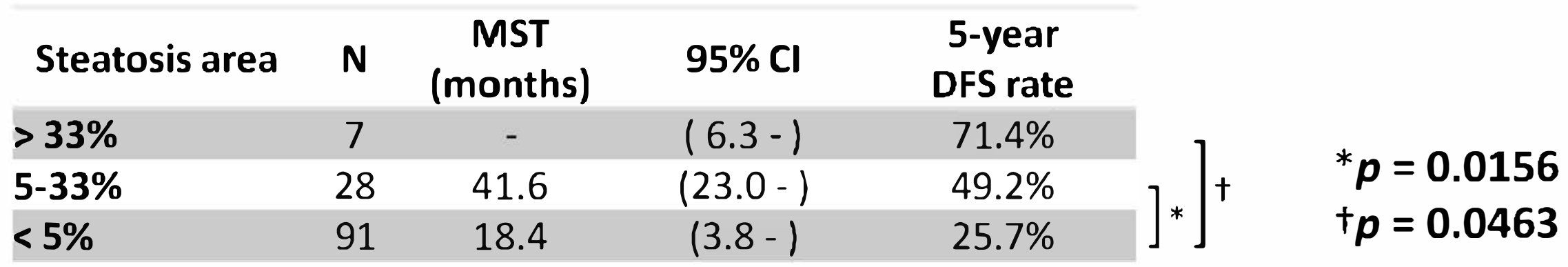




\section{LEGENDS OF SUPPLEMENTAL FIGURES}

Supplemental Figure 1 The components of NAFLD activity score of NAFLD-associated HCC patients.

Supplemental Figure 2 Comparisons of overall survival rates among the etiologies. The survival line of other known etiology cases was omitted. Median survival time (MST), 95\% CI, and overall survival rate at 5 years (5-year OS rate), are shown below the graph. Blanks in MST and the upper limit of 95\% CI represent unreached. All groups were compared using the log-rank test; the combinations of groups with $p<0.10$ were represented.

Supplemental Figure 3 Comparisons of disease-free survival rates among the etiologies in patients who underwent curative resection. The survival line of other known etiology cases was omitted. Median survival time (MST), 95\% CI, and disease-free survival rate at 5 years (5-year DFS rate) are shown below the graph. Blanks in MST and the upper limit of 95\% CI represent unreached. All groups were compared using the log-rank test; the combinations of groups with $p<0.10$ were represented.

Supplemental Figure 4 Comparison of disease-free survival rates according to the presence of liver cirrhosis in BC HCC patients who underwent curative resection. Median survival time (MST), 95\% CI, and disease-free survival rate at 5 years (5-year DFS rate) are shown below the graph.

Supplemental Figure 5 Comparison of disease-free survival rates according to the presence of liver cirrhosis in NBNC HCC patients who underwent curative resection. Median survival time (MST), 95\% CI, and disease-free survival rate at 5 years (5-year DFS rate) are shown below the graph.

Supplemental Figure 6 Comparison of disease-free survival rates according to the presence of 
steatosis in NBNC HCC patients who underwent curative resection. Median survival time (MST), 95\% CI, and disease-free survival rate at 5 years (5-year DFS rate) are shown below the graph.

Supplemental Figure 7 Comparison of disease-free survival rates according to the presence of steatosis in BC HCC patients who underwent curative resection. Median survival time (MST), 95\% CI, and disease-free survival rate at 5 years (5-year DFS rate) are shown below the graph. 
Supplemental Figure 1

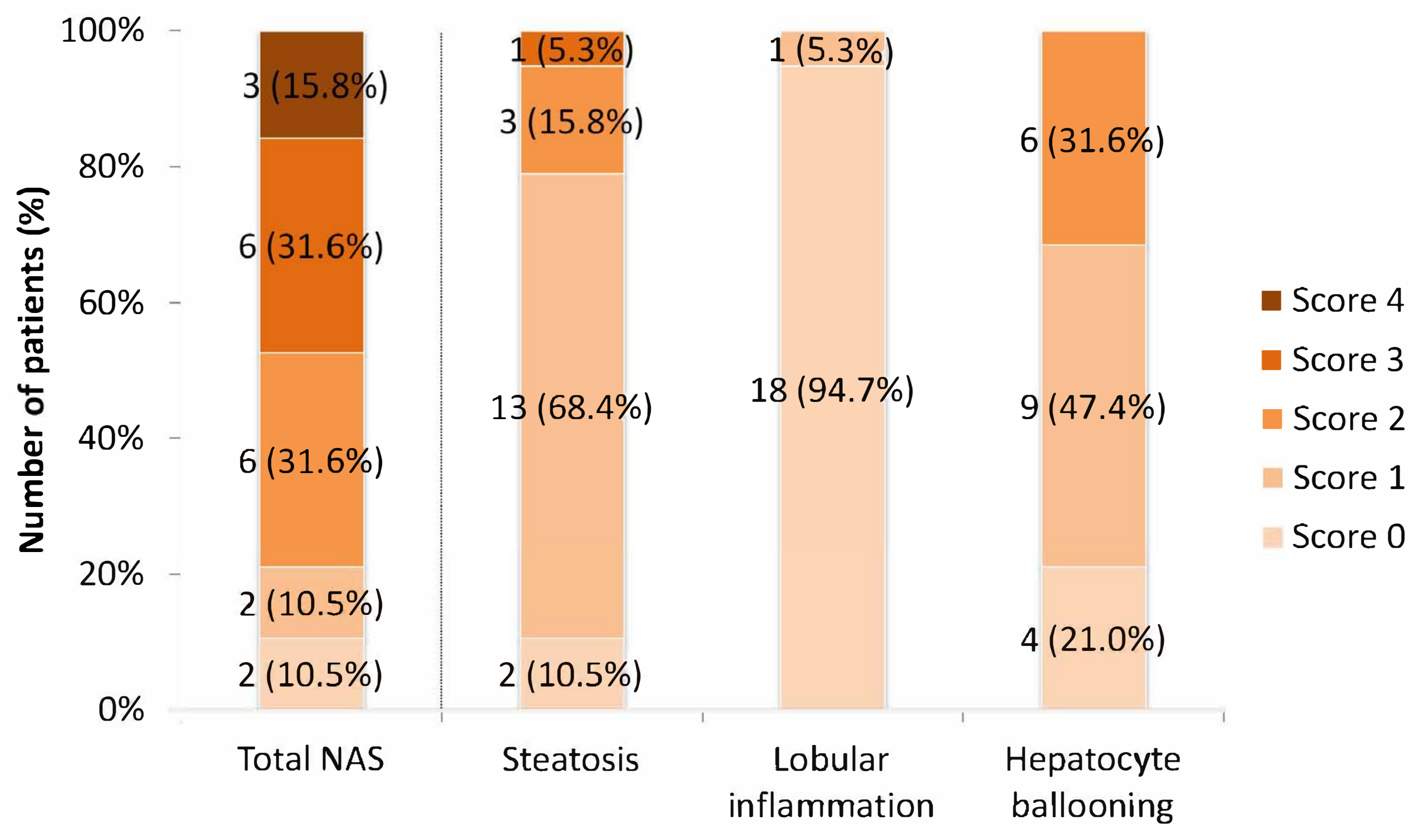




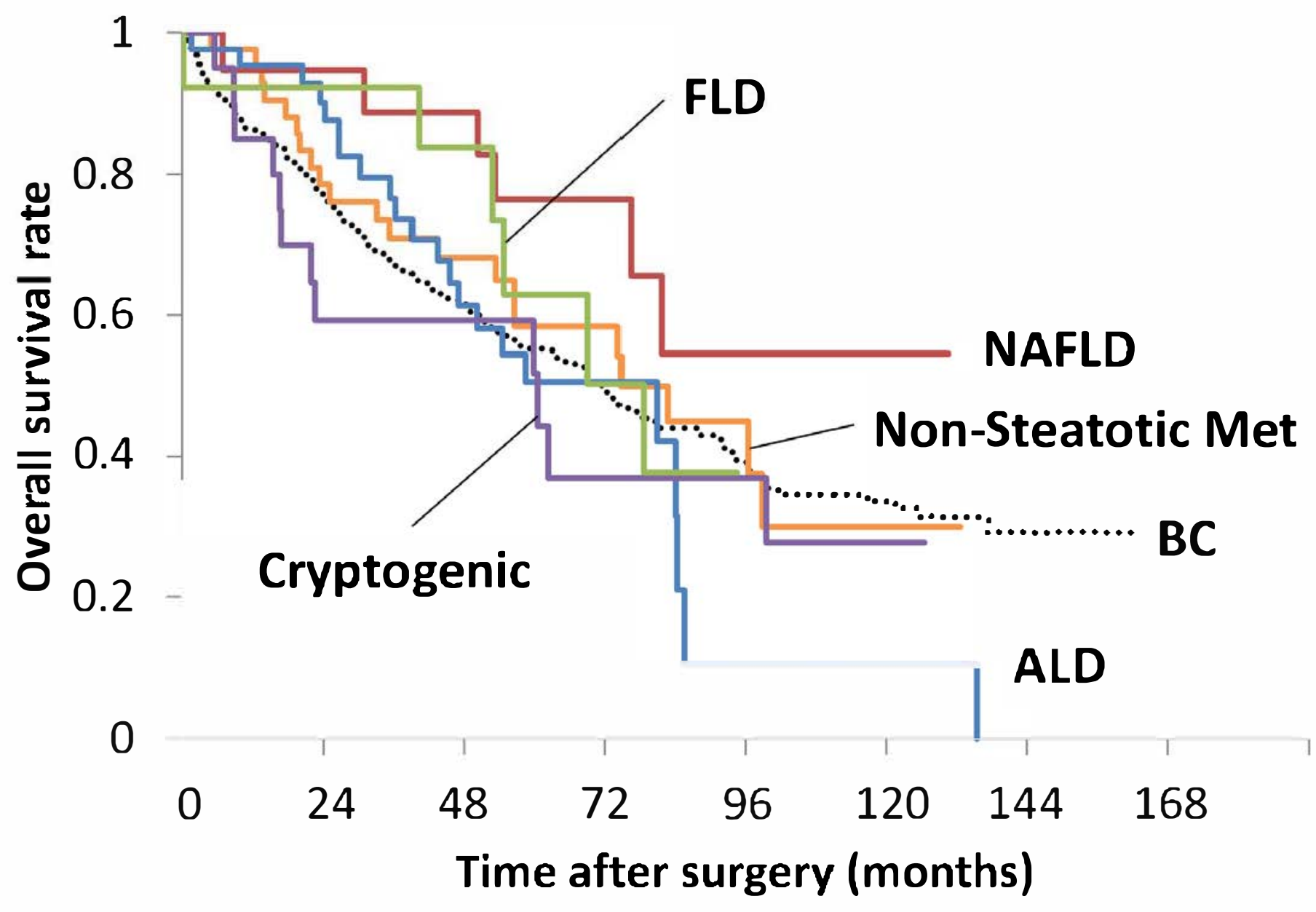

\begin{tabular}{|c|c|c|c|c|c|c|}
\hline & $\mathbf{N}$ & $\begin{array}{c}\text { MST } \\
\text { (months) }\end{array}$ & $95 \% \mathrm{Cl}$ & $\begin{array}{l}5 \text {-year } \\
\text { OS rate }\end{array}$ & \multirow[b]{2}{*}{]$_{*}$} & \\
\hline ALD & 43 & 80.9 & $(43.4-84.3)$ & $50.6 \%$ & & \multirow{6}{*}{$\begin{aligned}{ }^{*} \boldsymbol{p} & =\mathbf{0 . 0 3 9 6} \\
\dagger p & =0.0549 \\
\ddagger p & =0.0837\end{aligned}$} \\
\hline NAFLD & 19 & - & $(53.2-)$ & $76.5 \%$ & \multirow{5}{*}{${ }^{\dagger} \neq$} & \\
\hline FLD & 13 & 78.5 & $(40.2-)$ & $62.9 \%$ & & \\
\hline Non-Steatotic Met & 43 & 82.7 & $(53.4-)$ & $58.4 \%$ & & \\
\hline Cryptogenic & 21 & 59.9 & $(16.7-99.5)$ & $49.1 \%$ & & \\
\hline$B C$ & 373 & 72.4 & $(58.4-81.4)$ & $55.3 \%$ & & \\
\hline
\end{tabular}



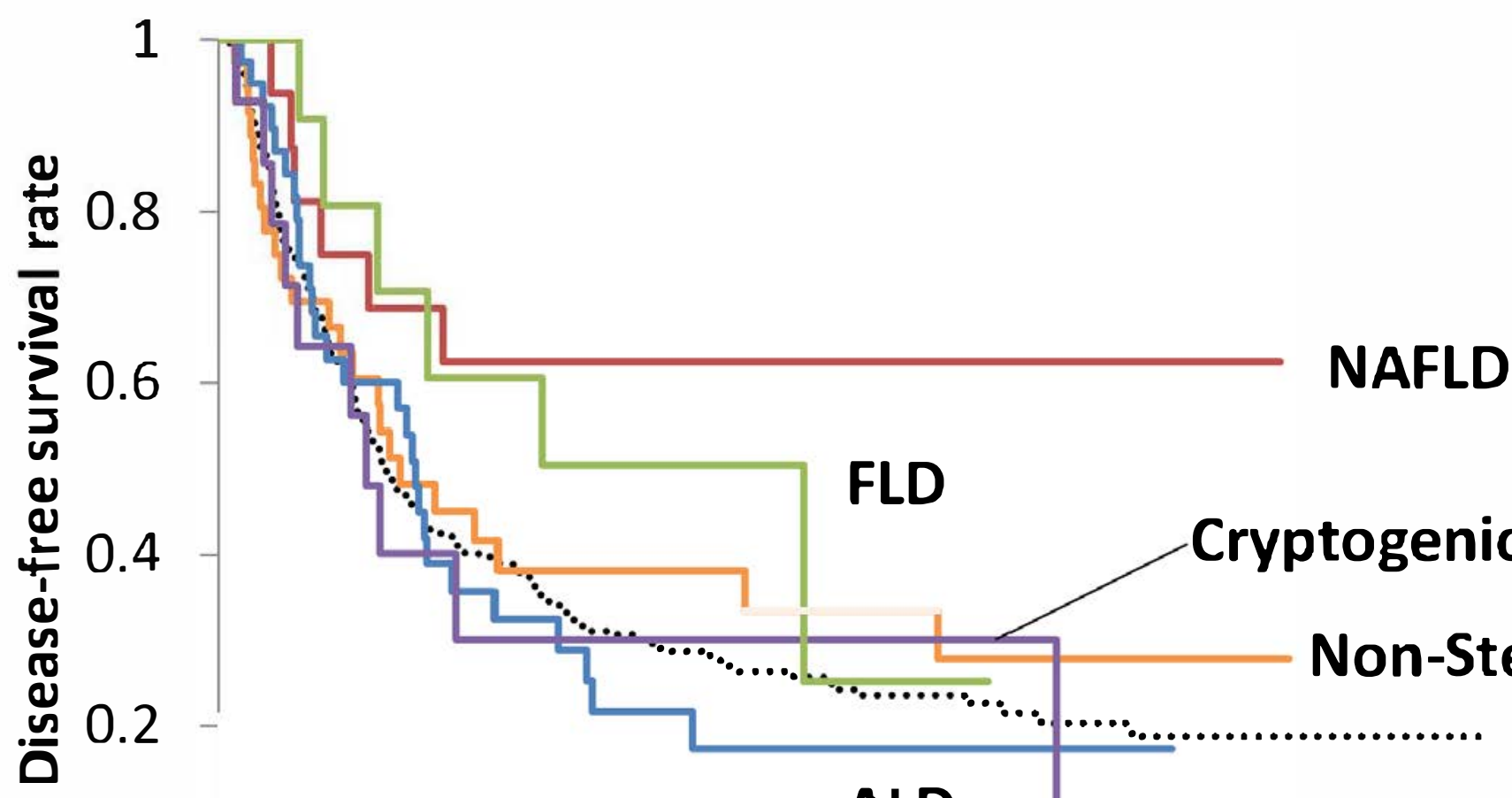

0
$0 \quad 24$
48
72
96
120
144
Time after surgery (months)

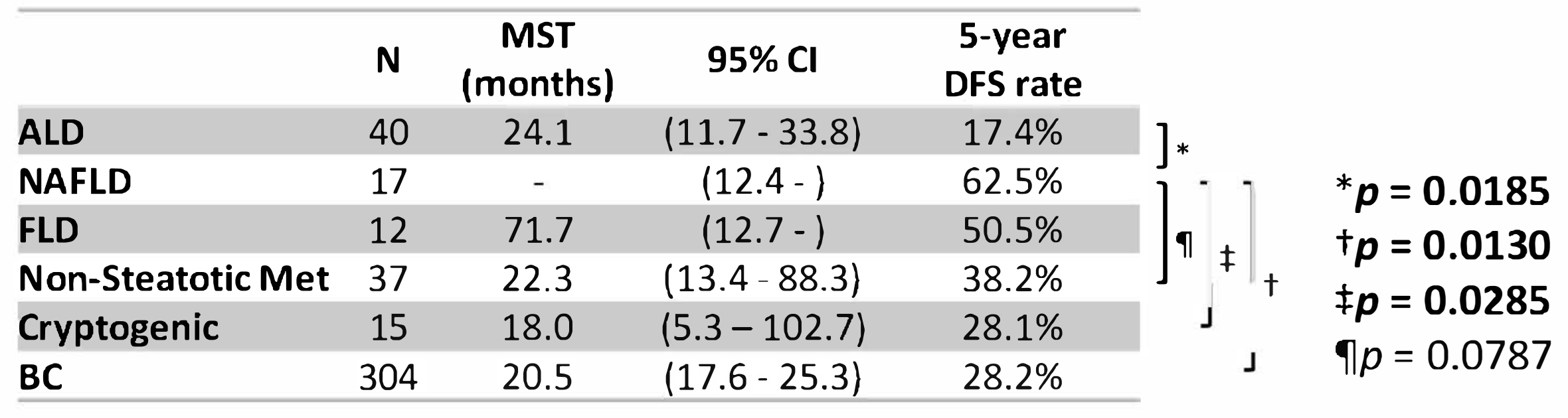




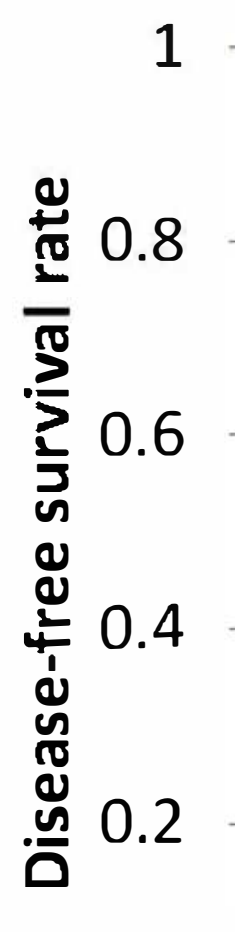

\section{BC HCC}

\section{Cirrhosis +/-}

Cirrhosis (-)

Cirrhosis (+)

0

$\begin{array}{lllllll}0 & 24 & 48 & 72 & 96 & 120 & 144 \\ & & & \text { Time after surgery (months) }\end{array}$

$\left.\begin{array}{ccccc}\text { Cirrhosis } & \mathbf{n} & \begin{array}{c}\text { MST } \\ \text { (months) }\end{array} & \mathbf{9 5 \% \mathrm { Cl }} & \begin{array}{c}\text { 5-year } \\ \text { DFS rate }\end{array} \\ + & 126 & 53.5 & (45.2-73.4) & 22.4 \% \\ - & 178 & 92.1 & (67.9-99.9) & 32.2 \%\end{array}\right] \boldsymbol{p}=\mathbf{0 . 0 0 4 8}$




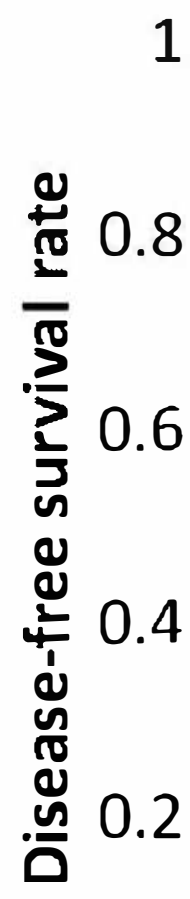

1

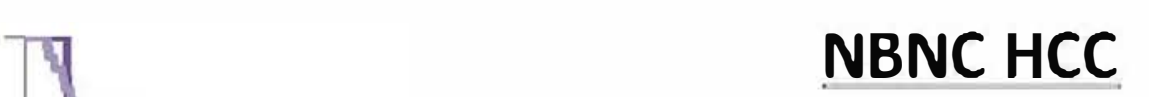

NBNC HCC

Cirrhosis +/-

Cirrhosis (-)

0

$0 \quad 24$

48

72

96

120

Time after surgery (months)

$\left.\begin{array}{ccccc}\text { Cirrhosis } & \mathbf{n} & \begin{array}{c}\text { MST } \\ \text { (months) }\end{array} & \mathbf{9 5 \%} \mathbf{C l} & \begin{array}{c}\text { 5-year } \\ \text { DFS rate }\end{array} \\ + & 25 & 24.5 & (13.0-71.7) & 31.7 \% \\ - & 101 & 24.1 & (16.3-39.6) & 34.6 \%\end{array}\right] p=0.9701$




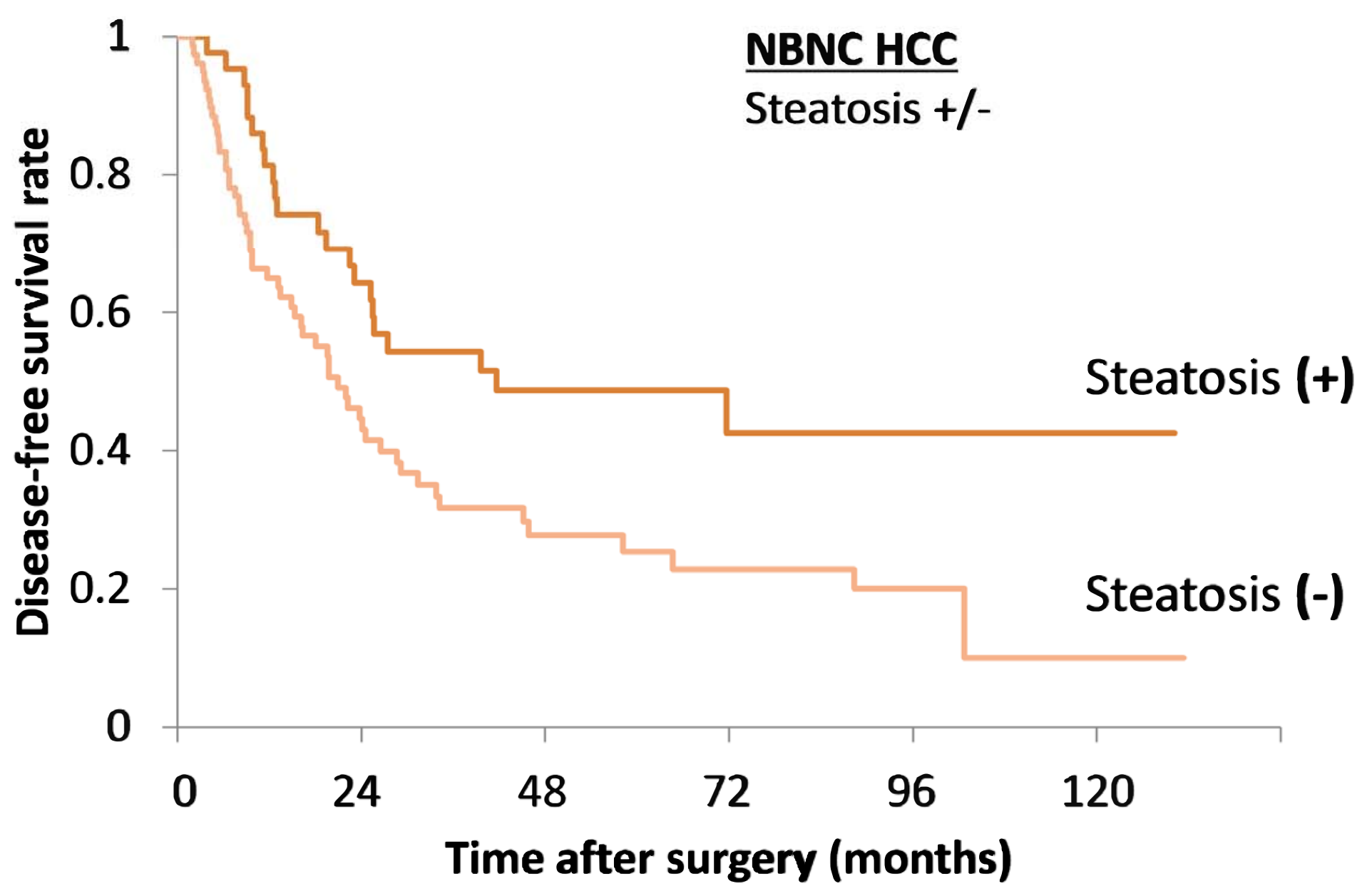

Supplemental Figure 6

\begin{tabular}{ccccc}
\hline Steatosis & $\mathbf{n}$ & $\begin{array}{c}\text { MST } \\
\text { (months) }\end{array}$ & $95 \% \mathrm{Cl}$ & $\begin{array}{c}\text { 5-year } \\
\text { DFS rate }\end{array}$ \\
\hline+ & 45 & 41.6 & $(23.0-)$ & $48.7 \%$ \\
- & 81 & 20.9 & $(13.4-28.6)$ & $25.2 \%$ \\
\hline
\end{tabular}




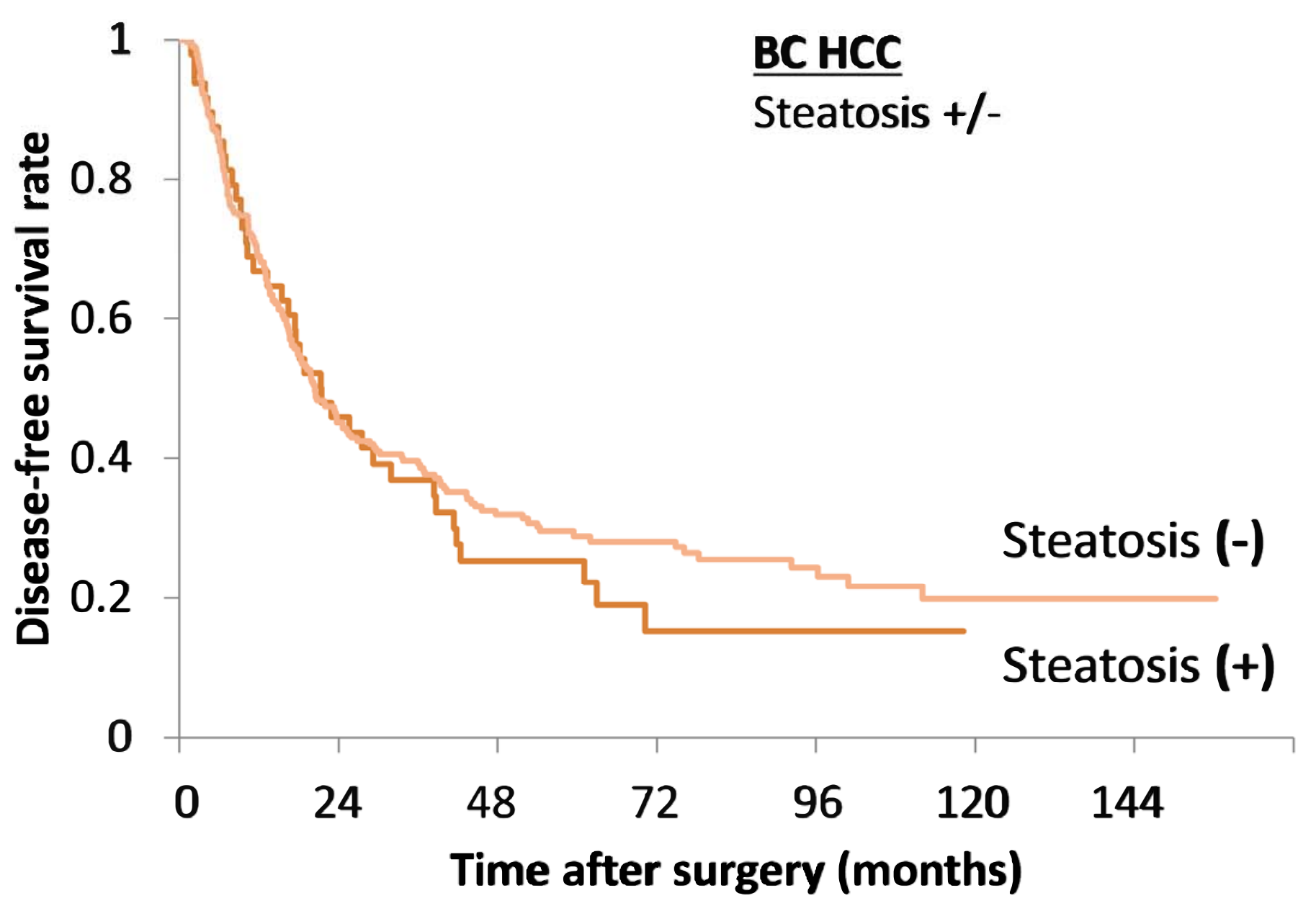

BCHCC 
Supplemental Table 1 Clinicopathologic characteristics of each subdivision of the NBNC HCC group

\begin{tabular}{|c|c|c|c|c|c|c|c|c|c|c|c|}
\hline & & \multicolumn{2}{|c|}{$\operatorname{ALD}(\mathrm{n}=43)$} & \multicolumn{2}{|c|}{ NAFLD $(\mathrm{n}=19)$} & \multicolumn{2}{|c|}{ FLD $(\mathrm{n}=13)$} & \multicolumn{2}{|c|}{ Non-Steatotic Met $(n=43)$} & \multicolumn{2}{|c|}{ Cryptogenic $(\mathrm{n}=21)$} \\
\hline \multicolumn{12}{|l|}{ Clinical backgrounds } \\
\hline \multirow[t]{2}{*}{ Gender } & Male & 43 & $(100.0 \%)+\rrbracket \S$ & 15 & $(79.0 \%) * \ddagger$ & 13 & $(100.0 \%) \dagger \uparrow \S$ & 33 & $(76.7 \%) * \ddagger$ & 13 & $(61.9 \%) * \ddagger$ \\
\hline & Female & 0 & $(0.0 \%)$ & 4 & $(21.0 \%)$ & 0 & $(0.0 \%)$ & 10 & $(23.3 \%)$ & 8 & $(38.1 \%)$ \\
\hline Age, yr & & 68 & \pm 7.39 & 69 & $\pm 8.3 \S$ & 69 & \pm 8.9 & 72 & $\pm 8.9 * \S$ & 62 & $\pm 11.8+9$ \\
\hline BMI, kg/ m² & & 23.3 & $\pm 3.1 \dagger \ddagger \S$ & 25.9 & $\pm 3.4 * \llbracket \S$ & 26.9 & $\pm 4.9 * \llbracket \S$ & 23.4 & $\pm 3.3 \dagger \ddagger \S$ & 20.7 & $\pm 2.7 * \dagger \neq \emptyset$ \\
\hline Obesity, + & & 10 & $(23.3 \%) \dagger \ddagger \S$ & 10 & $(52.6 \%) * \S$ & 7 & $(53.9 \%) * \S$ & 14 & $(32.6 \%) \S$ & 0 & $(0.0 \%) * \dagger \ddagger \emptyset$ \\
\hline Diabetes, + & & 19 & $(44.2 \%) \S$ & 12 & $(63.2 \%) \S$ & 5 & $(38.5 \%) \S$ & 23 & $(53.5 \%) \S$ & 0 & $(0.0 \%) * \dagger \ddagger \emptyset$ \\
\hline Dyslipidemia, + & & 9 & $(20.9 \%) \S$ & 3 & $(15.8 \%) \S$ & 3 & $(23.1 \%) \S$ & 4 & $(9.3 \%)$ & 0 & $(0.0 \%) * \dagger \ddagger$ \\
\hline Hypertension, + & & 20 & $(46.5 \%)$ ๆ§ & 9 & $(47.4 \%) \S$ & 7 & $(53.9 \%) \S$ & 29 & $(67.4 \%) * \S$ & 0 & $(0.0 \%) * \dagger \ddagger \rrbracket$ \\
\hline Ethanol intake $>70 \mathrm{~g} /$ day & & 43 & $(100.0 \%) \dagger \mp \rrbracket \S$ & 0 & $(0.0 \%) *$ & 0 & $(0.0 \%) *$ & 0 & $(0.0 \%) *$ & 0 & $(0.0 \%) *$ \\
\hline Ethanol intake $<20$ g/day & & 0 & $(0.0 \%)+\llbracket \S$ & 19 & $(100.0 \%) * \ddagger \rrbracket \S$ & 0 & $(0.0 \%) \dagger \uparrow \S$ & 36 & $(83.7 \%) * \dagger \ddagger$ & 14 & $(70.0 \%) * \dagger \neq$ \\
\hline \multicolumn{12}{|l|}{ Liver functions } \\
\hline ICG R15 & & 18.9 & $\pm 10.8 \S$ & 13.2 & \pm 7.1 & 14.4 & \pm 6.6 & 14.4 & \pm 6.8 & 11.7 & $\pm 6.2 *$ \\
\hline \multirow[t]{2}{*}{ Child-Pugh classification } & A & 40 & (93.0\%) & 18 & $(94.7 \%)$ & 12 & $(92.3 \%)$ & 41 & $(95.4 \%)$ & 21 & $(100.0 \%)$ \\
\hline & B & 3 & $(7.0 \%)$ & 1 & $(5.3 \%)$ & 1 & $(7.7 \%)$ & 2 & $(4.6 \%)$ & 0 & $(0.0 \%)$ \\
\hline \multicolumn{12}{|l|}{ Tumor characteristics } \\
\hline AFP, ng/ml & & 4984.4 & $\pm 21425 \S$ & 420.8 & \pm 1337 § & 878.1 & \pm 2772 & 8516.9 & \pm 29340 & 11400.1 & $\pm 40264 * \dagger$ \\
\hline $\mathrm{DCP}, \mathrm{mAU} / \mathrm{ml}$ & & 14544.0 & \pm 37218 & 6581.9 & \pm 11083 & 906.0 & \pm 1277 § & 7492.4 & $\pm 18006 \S$ & 30861.0 & \pm 62991 片 \\
\hline Tumor size, cm & & 5.5 & \pm 3.9 ๆ§ & 5.2 & \pm 2.3 ఇ§ & 4.5 & \pm 2.4 q§ & 7.9 & $\pm 4.3 * \dagger \neq$ & 9.7 & $\pm 5.4 * \dagger \ddagger$ \\
\hline \multirow[t]{2}{*}{ Number of tumors } & Solitary & 21 & $(48.8 \%) \S$ & 13 & (68.4\%) & 7 & (53.9\%) & 29 & $(67.4 \%)$ & 16 & $(76.2 \%) *$ \\
\hline & Multiple & 22 & $(51.2 \%)$ & 6 & (31.6\%) & 6 & (46.1\%) & 14 & (32.6\%) & 5 & $(23.8 \%)$ \\
\hline
\end{tabular}




\begin{tabular}{|c|c|c|c|c|c|c|c|c|c|c|c|}
\hline \multirow{3}{*}{ Tumor differentiation } & Well & 4 & $(9.7 \%) \S$ & 0 & $(0.0 \%)$ & 1 & $(7.7 \%)$ & 5 & $(11.9 \%) \S$ & 0 & $(0.0 \%) * \boldsymbol{q}$ \\
\hline & Moderate & 30 & $(73.2 \%)$ & 16 & $(84.2 \%)$ & 10 & $(76.9 \%)$ & 30 & (71.4\%) & 12 & $(57.1 \%)$ \\
\hline & Poor & 7 & $(17.1 \%)$ & 3 & $(15.8 \%)$ & 2 & (15.4\%) & 7 & $(16.7 \%)$ & 9 & (42.9\%) \\
\hline \multicolumn{2}{|l|}{ Microvascular invasion, + } & 18 & $(41.9 \%)$ & 7 & $(36.8 \%)$ & 4 & (30.8\%) & 14 & $(32.6 \%) \S$ & 13 & (61.9\%) ๆ \\
\hline \multirow[t]{2}{*}{ TNM Stage } & I, II & 32 & $(74.4 \%)$ & 13 & $(68.4 \%)$ & 11 & $(84.6 \%) \S$ & 27 & $(62.8 \%)$ & 11 & $(52.4 \%) \ddagger$ \\
\hline & III, IV & 11 & $(25.6 \%)$ & 6 & $(31.6 \%)$ & 2 & $(15.4 \%)$ & 16 & $(37.2 \%)$ & 10 & $(47.6 \%)$ \\
\hline \multicolumn{12}{|c|}{ Underlying parenchymal changes } \\
\hline \multirow[t]{4}{*}{ Steatosis, +} & & 15 & $(34.9 \%) \dagger \ddagger \rrbracket \S$ & 19 & $(100.0 \%) * \llbracket \S$ & 13 & $(100.0 \%) * \llbracket \S$ & 0 & $(0.0 \%) * \dagger \ddagger$ & 0 & $(0.0 \%) * \dagger \ddagger$ \\
\hline & $<5 \%$ & 4 & $(9.3 \%)$ & 2 & $(10.5 \%)$ & 4 & (30.8\%) & 0 & $(0.0 \%)$ & 0 & $(0.0 \%)$ \\
\hline & $5-33 \%$ & 8 & (18.6\%) & 13 & (68.4\%) & 9 & (69.2\%) & 0 & $(0.0 \%)$ & 0 & $(0.0 \%)$ \\
\hline & $>33 \%$ & 3 & $(7.0 \%)$ & 4 & (21.1\%) & 0 & $(0.0 \%)$ & 0 & $(0.0 \%)$ & 0 & $(0.0 \%)$ \\
\hline Cirrhosis, + & & 13 & (30.2\%) & 2 & $(10.5 \%)$ & 3 & (23.1\%) & 7 & (16.3\%) & 4 & $(19.1 \%)$ \\
\hline
\end{tabular}

Data represent the mean \pm standard deviation or the number of patients.

NBNC HCC: non-B non-C hepatocellular carcinoma

ALD: alcoholic liver disease

NAFLD: nonalcoholic fatty liver disease

FLD: fatty liver disease

Non-Steatotic Met: non-steatotic and metabolic syndrome-associated 
BMI: body mass index

ICG R15: indocyanine green retention rate at 15 minutes

AFP: alpha-fetoprotein

DCP: des-gamma-carboxy prothrombin

*: $p<0.05$ vs. ALD, $\uparrow: p<0.05$ vs. NAFLD, $\ddagger: p<0.05$ vs. FLD, $\mathbb{~ : ~} p<0.05$ vs. Non-Steatotic Met, $\S: p<0.05$ vs. Cryptogenic 
Supplemental Table 2 Clinicopathologic characteristics of NAFLD associated-HCC patients according to the NAS category

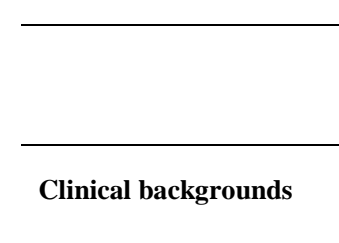

NAS $<3(n=10)$

NAS $\geq 3(n=9)$

$p$ value

Clinical backgrounds

Gender

Male

9

(90.0\%)

6

(66.7\%)

0.2062

Female

(10.0\%)

3

(33.3\%)

Age, yr

67.5

$\pm 10.1$

70.1

$\pm 6.0$

0.7741

BMI, kg/ m²

27.8

$\pm 3.5$

23.9

$\pm 1.7$

0.0062

Obesity, +

8

(80.0\%)

2

(22.2\%)

0.0094

Diabetes, +

6

(60.0\%)

6

(66.7\%)

0.7633

Dyslipidemia, +

1

(10.0\%)

2

(22.2\%)

0.4633

Hypertension, +

6

(60.0\%)

$3 \quad$ (33.3\%)

0.2419

Liver functions

ICG R15

$\pm 8.6$

11.7

$\pm 5.1$

0.5937 
A

Tumor characteristics

AFP, ng/m

DCP, $\mathrm{mAU} / \mathrm{ml}$

Tumor size, cm

Number of tumors

Solitary

Multiple

72.0

$\pm 169.3$

808.4

$+1915.4$

2084.3

$\pm 5055.6$

11579.3

$\pm 13936.6$

0.0792

4.8

$\pm 2.6$

5.7

(80.0\%)

5

(55.6\%)

(44.4\%)

Tumor differentiation

(20.0\%)

$0.0 \%)$

0.0237

Moderate

10

$(100.0 \%)$

$(66.7 \%)$

Poor

$(0.0 \%)$

(33.3\%)

Microvascular invasion, +

$(10.0 \%)$

6

$(66.7 \%)$

0.0079

TNM Stage

I, II

(90.0\%)

0.0279 

III, IV
(10.0\%)
5
(55.6\%)

Underlying parenchymal changes

Cirrhosis, +

Fibrosis stage

F0

F1

F2

F3

F4
(20.0\%)

(20.0\%)

(40.0\%)

(10.0\%)

(10.0\%)
(11.1\%)

0.9372

(11.1\%)

(33.3\%)

(22.2\%)

(22.2\%)

$11.1 \%)$

Data represent the mean \pm standard deviation or the number of patients.

NAFLD: nonalcoholic fatty liver disease

HCC: hepatocellular carcinoma

NAS: NAFLD activity score

BMI: body mass index 
ICG R15: indocyanine green retention rate at 15 minutes

AFP: alpha-fetoprotein

DCP: des-gamma-carboxy prothrombin 